\title{
Expression and localization of somatostatin receptor types 3, 4 and 5 in the wild-type, SSTR1 and SSTR1/SSTR2 knockout mouse cochlea
}

\author{
Vesna Radojevic • Daniel Bodmer
}

Received: 5 November 2013 / Accepted: 24 July 2014 / Published online: 23 August 2014

(C) Springer-Verlag Berlin Heidelberg 2014

\begin{abstract}
Somatostatin (SST) is a peptide hormone that exerts inhibitory effects mediated through binding to specific cell surface $\mathrm{G}$ protein-coupled receptors, of which five distinct subtypes (SSTR1-SSTR5) have been characterized. Our study performed on mouse cochlear hair cells shows the expression and localization of the three receptors (SSTR3-SSTR5) in wild-type (WT), single-knockout (SSTR1 KO) and doubleknockout SSTR1/SSTR2 (DKO) mice. Similar SSTRs expression were observed in the inner hair cells (IHC), outer hair cells (OHC) and supporting cells of cultivated P7 mouse organ of Corti (OC) explants as well as in cultivated cochlear neuroepithelial supporting cells (NEsc). We found differences in the expression of SSTR3-5 in WT, SSTR1 KO and DKO mouse cochlea, which might be explained as a compensatory effect in the cochlea after the loss of SSTR1 and/or SSTR2.
\end{abstract}

Keywords Cochlea $\cdot$ Hair cells $\cdot$ Knockout mice $\cdot$ Organ of Corti $\cdot$ Somatostatin $\cdot$ Somatostatin receptor

\section{Introduction}

SST, a regulatory peptide with two bioactive forms, SST-14 and SST-28, is produced in neuroendocrine cells in the brain and periphery and acts on a number of tissue targets to modulate neurotransmission, cell secretion and cell proliferation (Reichlin 1983; Epelbaum et al. 1994; Patel 1999). A family of transmembrane receptors mediates the physiological

\footnotetext{
V. Radojevic $(\bowtie) \cdot$ D. Bodmer

Department of Biomedicine, University Hospital Basel, Petersgraben 4, 4031 Basel, Switzerland

e-mail: vesna.radojevic@unibas.ch

V. Radojevic · D. Bodmer

Clinic for Otolaryngology, Head and Neck Surgery, University

Hospital Basel, Petersgraben 4, 4031 Basel, Switzerland
}

actions of SST. Five distinct SST receptor types (SSTR1-5) have been identified (Patel 1999).

The distribution of mRNA expression for all five SST receptor types has been investigated extensively using different techniques in rats and humans (Harrington et al. 1995; Kong et al. 1994; Schindler et al. 1995). Published studies are in agreement and suggest that all SSTR types are variably expressed widespread throughout the brain. In rats, SSTR1 mRNA is the most abundant, followed by SSTR2, SSTR5, SSTR3 and SSTR4 (Patel 1999; Breder et al. 1992; Kong et al. 1994; Thoss et al. 1995; Beaudet et al. 1995). SSTR3 immunoreactivity is widely distributed throughout the human brain and is particularly enriched in the cortex, hippocampus, amygdala, hypothalamus and cerebellum (Hervieu and Emson 1999; Schindler et al. 1995). Immunohistochemistry of adult rat brain sections showed that SSTR4 is distributed in both the cell body and neuronal processes in the cerebral cortex, hippocampus and several nuclei of the brainstem (Selmer et al. 2000). Schreff et al. (2000) showed that SSTR4 is strictly somatodendritic and most likely functions in a postsynaptic manner. SSTR4 may have a previously unappreciated function during neuronal degeneration/regeneration. SSTR5 is prominently expressed in the basal forebrain, suggesting that it is involved in the mediation of the effects of SST on the sleep-wake cycle through its association with cortically projecting subcortical systems (Stroh et al. 1999). Most SSTR subtypes have been shown to modulate voltagegated $\mathrm{Ca}^{2+}$ channels and glutamate receptor channels in several organ systems (Cervia and Bagnoli 2007). Excessive $\mathrm{Ca}^{2+}$ influx into cells is thought to be a major contributor to cell death in ischemic and excitotoxic models of neuronal injury (Smaili et al. 2009). SSTRs are also involved in the regulation of ion channels, the inhibition of $\mathrm{Ca}^{2+}$ channels and activation of $\mathrm{K}^{+}$ channels, involved in the release of several neurotransmitters and the modulation of neurotransmission (Patel 1999; Petrucci et al. 2001; Johnson et al. 2001). All five subtypes of SSTRs are expressed in the mammalian retina and are distributed to specific 
cell populations, including subtypes of photoreceptor, bipolar, amacrine and ganglion cells (Thermos 2003; Ke and Zhong 2007; Vasilaki and Thermos 2009). Studies have shown that SST analogues may be effective therapeutic agents in retinal diseases (Vasilaki and Thermos 2009).

However, in contrast to the situation in the retina, little information is available on the expression or function of somatostatin and its receptors in the inner ear. Tachibana et al. (1979) reported on somatostatin-like immunoreactivity in the medial geniculate body, cochlear nucleus, inferior colliculus, auditory cortex and in the cochlea. Also, somatostatin-like immunoreactivity could be observed in the cochlear nuclei of postnatal rats and it was suggested that somatostatin might be important for the development of the auditory system (Takatsuki et al. 1982).

Our previous study demonstrated that SSTR1 and SSTR2 mRNA and protein are expressed in the mouse cochlea and localized in IHC and OHC and in supporting cells of the OC (Radojevic et al. 2011). The present study is the first attempt to determine SSTR3, -4 and -5 expression in the cochlea from WT and DKO mice.

\section{Materials and methods}

\section{Animals}

Experiments were performed on mouse cochleae from WT C57BL/6 mice of both sexes. The patterns of SSTR3-5 expression were also observed at postnatal ages $(n=10$ per postnatal day for mRNA isolation). Immunohistochemistry was performed on four mice per postnatal day. The procedures used to generate SSTR $1^{-1-}$ (homozygous SSTR1 KO)/ C57BL6J mice (Kreienkamp et al. 1999) and SSTR2 ${ }^{-/-}$(homozygous SSTR2 KO)/C57BL6J mice have been described previously (Zheng et al. 1997). SSTR1 KO mice were crossed with SSTR2 KO mice to produce DKO mice. Age-matched WT mice were produced from C57BL6J mice used to stabilize the genetic backgrounds of SSTR1 KO and SSTR2 KO mice (Bodmer et al. 2012).

Animal care and use were conducted in conformity with the European Communities Council Directive of 24 November 1986 (86/609/EEC) and reviewed and permitted by the Kantonales Veterinäramt, Basel, Switzerland.

Tissue extraction and culture

The mice were decapitated and cochlear microdissections were performed under a light microscope to isolate the $\mathrm{OC}$ and the spiral ganglion (SG). Brain tissue was removed from the same animals. During microdissection, the different tissues were maintained in ice-cold PBS.

\section{RNA extraction}

The SG, brain (Br) and cell lysate (Jayanthi et al. 2005) from P7 mice, the OC from WT mouse pups (P0-P21) and OC of DKO mouse pups by age P14 and P21 were placed separately in RNAlater (Qiagen, Hombrechtikon, Switzerland). RNA isolation of the brains and inner ear components was performed using the RNAeasy Minikit (Qiagen) and an UltraTurrax T8 tissue homogenizer (IKA-Werke, Staufen, Germany) according to the manufacturer's instructions, including DNase treatment. The quantity and quality of isolated RNA was determined with a NanoDrop ND 1000 (NanoDrop Technologies, DE, USA). The 260-/280-nm ratio of all samples was between 1.8 and 2.1.

Reverse transcription polymerase chain reaction

Total RNA (500 ng) was reverse transcribed into cDNA using the first strand cDNA synthesis kit for reverse transcription polymerase chain reaction (RT-PCR) (Roche Applied Bioscience, Rotkreuz, Switzerland) according to the supplier's instructions. PCR was performed using the PCR Master Mix (Roche Applied Bioscience) with primers specific for SSTR3, SSTR4 and SSTR5. $\beta$-actin primers were used as a positive control for cDNA synthesis. The primers were designed to cross at least one exon junction for the specific amplification of cDNA and to recognize contamination with genomic DNA. The primer sequences were:

SSTR1 forward (fwd): 5'-CAGGGTAGCCATTCTC ATC-3',

SSTR1 reverse (rev): 5'-AGCGTGGAAGTGACCAGA AAG-3', SSTR2 (fwd): 5'-CGCATGGTGTCCATCGTAGT-3', SSTR2 (rev): 5'-GGATTGTGAATTGTCTGCCTTGA-3', SSTR3 (fwd): 5'-CTGGCTGTCAGTGGCATCTT-3', SSTR3 (rev): 5'-CACATGAGATCCGAAGGGC-3', SSTR4 fwd: 5'-CAGCGGCATGGTCACTATC-3', SSTR4 rev: 5'-CCGTCCACGCTAAGCACTG-3', SSTR5 fwd: 5'-TTCCTGGCAACGCAGAATG-3', SSTR5 rev: 5'-CAAGAGCGGCAGAGACATGAG-3', $\beta$-actin fwd, 5'-ACGGTCAGGTCATCACTATCGG CA-3',

$\beta$-actin rev, 5'-ATCCTGTCAGCAATGCCTGGGT-3' (Microsynth, St. Gallen, Switzerland).

PCR reactions were performed in an Eppendorf Mastercycler (Eppendorf, Hamburg, Germany). For SSTR3, SSTR4 and SSTR5, an initial denaturing step at $94{ }^{\circ} \mathrm{C}$ for $30 \mathrm{~s}$ was followed by $30 \mathrm{PCR}$ cycles consisting in denaturation at $94{ }^{\circ} \mathrm{C}$ for $15 \mathrm{~s}$, annealing at $60{ }^{\circ} \mathrm{C}$ for $30 \mathrm{~s}$ and extension at $72{ }^{\circ} \mathrm{C}$ for $15 \mathrm{~s}$. The PCR was completed with a final extension step at $72{ }^{\circ} \mathrm{C}$ for $3 \mathrm{~min}$. $\beta$-actin was subjected to $30 \mathrm{PCR}$ 
cycles consisting in denaturation at $94{ }^{\circ} \mathrm{C}$ for $30 \mathrm{~s}$, annealing at $58^{\circ} \mathrm{C}$ for $45 \mathrm{~s}$ and extension at $72^{\circ} \mathrm{C}$ for $90 \mathrm{~s}$. The PCR was completed with a final extension step at $72^{\circ}$ for $5 \mathrm{~min}$. PCR products were stained with SYBR Green I (Molecular Probes, OR, USA), separated by electrophoresis on a $2 \%$ agarose gel and visualized under ultraviolet light. Omission of RT-PCR was used as the no template control.

Real-time PCR

Total RNA (500 ng) was reverse transcribed into cDNA using the First Strand cDNA synthesis kit (Roche Applied Biosciences, Foster City, USA) according to the manufacturer's instructions. The reaction occurred in an ABI Prism 7900HT Sequence Detection System (Applied Biosystems) using Fast Start Universal SYBR Green Master (Rox; Roche Applied Biosciences). The primer sequences were:

\section{SSTR3-fwd: 5'-TGTTACCTATCCCTCATCCGAG-3',} SSTR3-rev: 5'-GAGAGATCAAGATGCCACTGAC-3', SSTR4-fwd: 5'-AGCTGGGCATGGTCACTATC-3', SSTR4-rev: 5'-CAGACACACGAGCGCATAGA -3'

Fig. 1 Immunolabeling of SST3, -4 and -5 receptors in the mouse cochlea at E14 and E17. SSTRs are in red and transcription factor Hes6 is in green $(\mathbf{a}, \mathbf{b}, \mathbf{e})$. In the immunofluorescence images at E14, SSTR3, -4 and -5 can be observed in Kölliker's organ $(\mathrm{KlO})$, in epithelial cells located in the embryonic cochlear duct (b, c, d). In immunofluorescence images at E17, SSTR4 and -5 can be observed in the embryonic OHC and IHC. Weak labeling was observed in supporting cells of the KoD. SSRT3 at age of E17 was not observed in $\mathrm{HC}$.

Transcription factor Hes5 staining was used as a marker for the embryonic cochlea. Primary antibody omission was used as negative control. Scale bar $50 \mu \mathrm{m}$

\section{SSTR5-fwd: 5'-GGCTTCCACACCTAGCTGG-3',} SSTR5-rev: 5'-AGCACAGGCACTAATACCGC3'(Microsynth).

Each reaction contained $300 \mathrm{nM}$ of primer. The reaction occurred at $95^{\circ} \mathrm{C}$ for $10 \mathrm{~min}$, followed by 40 cycles of $95^{\circ} \mathrm{C}$ for $15 \mathrm{~s}$ and $60{ }^{\circ} \mathrm{C}$ for $60 \mathrm{~s}$. We calculated the relative quantities of specifically amplified cDNA using the comparative threshold cycle method. GAPDH was used as an endogenous reference (Microsynth) (Bodmer et al. 2012; Hall et al. 2007; Chen and Ruan 2009). Template-free and reverse transcription-free controls excluded non-specific amplification and DNA contamination.

Preparation of paraffin cochlear sections

The mice were killed with an overdose of sodium pentobarbital $(100 \mathrm{mg} / \mathrm{kg})$ and transcardially perfused with $50 \mathrm{ml}$ of phosphate-buffered $4 \%$ paraformaldehyde $\left(\mathrm{pH} \mathrm{7.4,} 4{ }^{\circ} \mathrm{C}\right)$. The inner ear was carefully removed and decalcification carried out in a light-protected flask for 10 days in a solution of
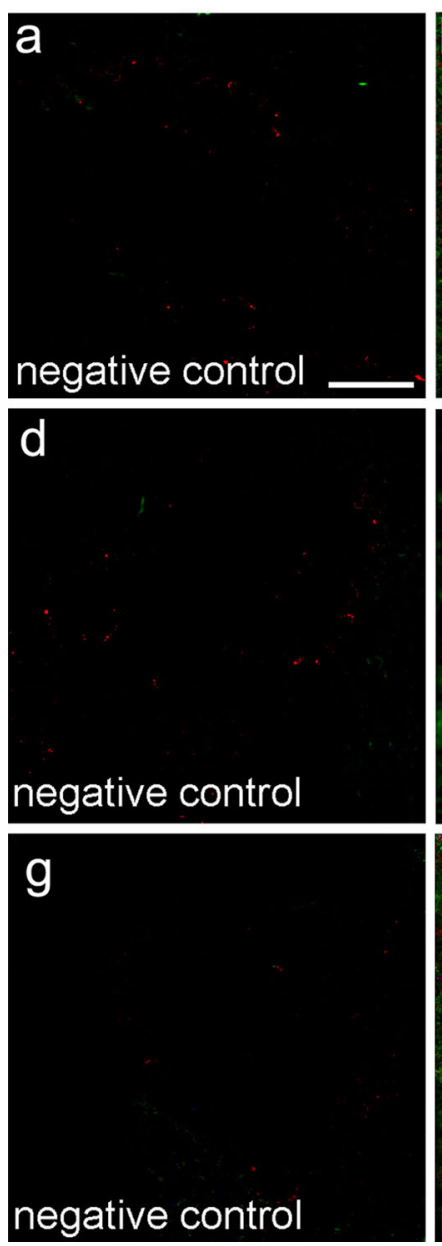

E14
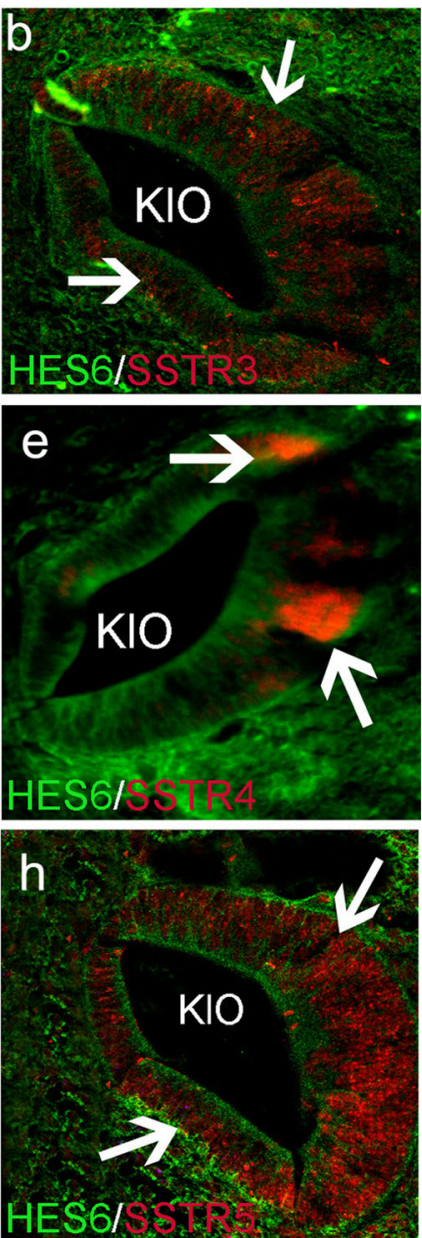

E17
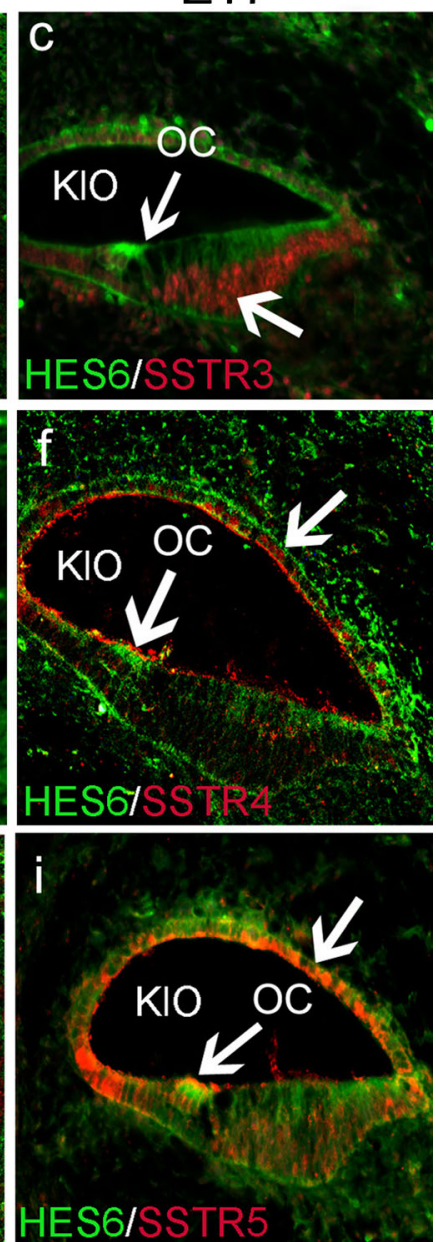
120 mM EDTA (Merck, NJ, USA) in distilled water (pH 6.8). The cochleae were then prepared for paraffin embedding by dehydration in graded ethanol solutions $(70,80,95 \%$ and $3 \times$ $100 \%$, each for $1 \mathrm{~h})$, xylol $(3 \times$ for $1 \mathrm{~h})$ and paraplast $(2 \times$ at $-60{ }^{\circ} \mathrm{C}$ for $1 \mathrm{~h}$ and $1 \times$ at $-60^{\circ} \mathrm{C}$ for $10 \mathrm{~h}$ ). The samples were embedded in paraffin at $56^{\circ} \mathrm{C}$.

Histology and immunocytochemistry

For histological evaluation, the head of embryos and cochlear sections were cut 8 - $\mu \mathrm{m}$-thick on a Leitz microtome and mounted on Superfrost plus slides (Menzel, Braunschweig, Germany). Sections were deparaffinized, rehydrated, washed in PBS for 5 min and subjected to immunohistochemistry. The NEsc attached to poly-lysine-coated cover slips were fixed in $4 \%$ paraformaldehyde for $15 \mathrm{~min}$, washed twice and kept in 0,01 M PBS at $4{ }^{\circ} \mathrm{C}$ for further processing. Microtome sections and cell cultures were incubated for $1 \mathrm{~h}$ at room temperature in blocking solution containing TBS ( $50 \mathrm{mM}$ Tris, $0.9 \% \mathrm{NaCl}), 0.5 \%$ Triton $\mathrm{X}-100(\mathrm{pH} 7.35)$ and $3 \%$ normal goat serum (NGS). The sections were incubated with primary rabbit polyclonal antiSSTR3, anti-SSTR4, anti-SSTR5 (1:400; AbCam, UK), mouse monoclonal anti-Hes5 antibody (1:300; AbCam) and mouse monoclonal antibody against myosin VIIa (1:500; Abcam). All antibodies were diluted in TBS with $1 \%$ NGS overnight at $4{ }^{\circ} \mathrm{C}$. After three washes in TBS, the sections were incubated for $1 \mathrm{~h}$ at room temperature with the appropriate Alexa-conjugated secondary antibodies (1:250; Molecular Probes, Lubio Science, Switzerland) diluted in TBS with $1 \%$ NGS for $2 \mathrm{~h}$ at room temperature. After washing in TBS, the sections were counterstained with DAPI and mounted on glass slides with Mowiol.

Slices were visualized on an Olympus AX-70 microscope equipped with a spot digital camera. The recorded images were adjusted for brightness and contrast using Image-Pro Plus and Photoshop image processing software.

\section{Western blot}

Animals at P21 were decapitated and cochleae were carefully micro-dissected in ice-cold PBS. The OC was separated from the $\mathrm{SG}$ and the stria vascularis (SV). Brain extract was used as a positive control. Explants were homogenized in CelLytic buffer containing protease inhibitor cocktail (Sigma-Aldrich, St. Louis, USA) and centrifuged. Supernatants were aspirated and placed in a new tube. Protein concentration was determined using the BCA Protein Assay Reagent kit (Pierce, Rockford, USA) according to the manufacturer's instructions. Lysates were mixed with Laemmli sample buffer and heated at $95{ }^{\circ} \mathrm{C}$ for $5 \mathrm{~min}$. Then, $20 \mu \mathrm{g}$ of each lysate was resolved on a $10 \%$ SDS-PAGE gel and blotted onto a polyvinylidene fluoride membrane. The non-specific sites of the transferred proteins were blocked with blocking solution (Roche) diluted (1:100) in PBS-T (1.54 mM KH 2 PO4; $155.17 \mathrm{mM} \mathrm{NaCl} ; 2.71 \mathrm{mM}$
$\mathrm{Na}_{2} \mathrm{HPO} 4-7 \mathrm{H}_{2} \mathrm{O}, \mathrm{pH} 7.2 ; 0.1 \%$ Tween-20) for $1 \mathrm{~h}$ at room temperature. The membrane was washed with PBS-T $(3 \times$ $10 \mathrm{~min}$ ) and then incubated with primary antibodies in TBS. The following primary antibodies were used: rabbit polyclonal anti-SSTR3, anti-SSTR4 and anti-SSTR5 antibodies (1:1000; Abcam) and mouse monoclonal anti- $\beta$-actin (1:5000, Santa Cruz Biotech, CA, USA). The membranes were incubated with the primary antibodies overnight at $4{ }^{\circ} \mathrm{C}$. After that, the blots were incubated with monoclonal anti-rabbit peroxidase-conjugated secondary antibody (1:80,000; Sigma-Aldrich, Switzerland) and anti-mouse peroxidase-conjugated secondary antibody (1:200,000; Sigma-Aldrich).

The blots were washed with PBS-T $(3 \times 10 \mathrm{~min})$ and incubated with appropriate POD-conjugated secondary antibody for $1 \mathrm{~h}$ at room temperature (Roche). After washing, bands were visualized using developmental solution BM Chemiluminiscence (Roche). Immunopositive bands were visualized by autoradiograpy. Serial exposures were made to radiographic film (Hyperfilm ECL; Amersham Biosciences).

\section{Statistical analysis and software}

Statistical analyses were performed using Student's $t$ test for unpaired samples. Means were considered significant when $p$ $<0.05$. The Origin computer program (Microcal Software, Northampton, MA, USA) was used for statistical analysis and to generate graphs. ANOVA test was used for comparison of multiple data, followed by the Bonferroni's post hoc test.
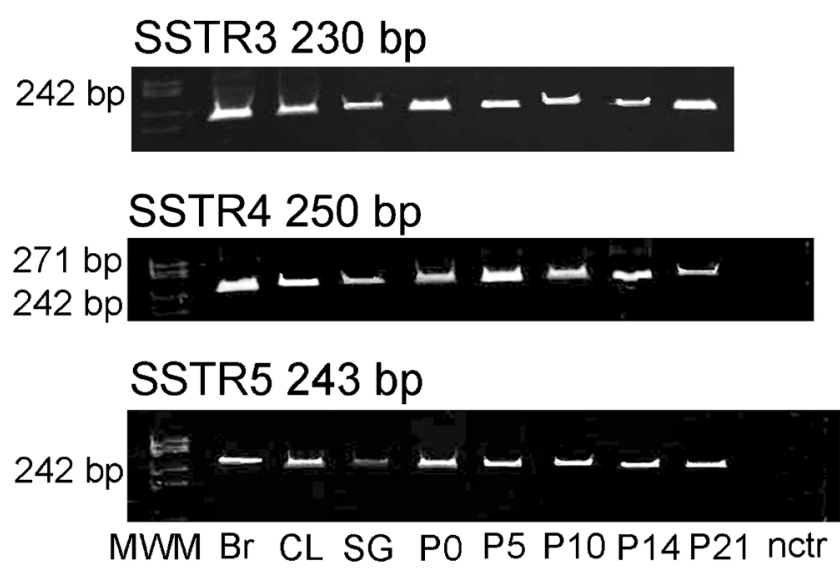

$190 \mathrm{bp}$

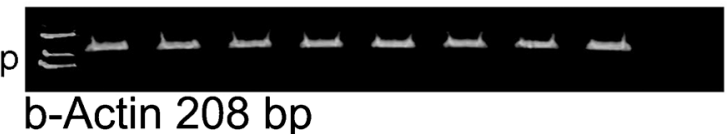

Fig. 2 Expression of SSTR3, -4 and -5 in the cell lysat (Jayanthi et al. 2005) of neuroepithelial cells, SG and in OC explants at different postnatal ages. All three mRNAs were detected in the OC of wild-type mice using specific primer sets at $\mathrm{P} 0, \mathrm{P} 5, \mathrm{P} 10, \mathrm{P} 14$ and $\mathrm{P} 21$. Brain $(\mathrm{Br})$ RNA was used as a positive control. Omission of RT-PCR was used as the no template control (Blatchley et al. 1987). $\beta$-actin was used to control for cDNA synthesis. $M W M$ Molecular weight marker 


\section{Results}

Embryonic expression of SSTR3, 4 and 5

At E14, all three SSTRs appeared to be associated with the thickened epithelial cells located in Kölliker's organ (Fig. 1b, e, h). SSTR4 and SSTR5 immunoreactivity was clearly detected at E17 in the region of hair cells and supporting cells along the length of the cochlear duct, which in this stage have become arranged into the characteristic patten for the OC (Fig. 1c, f, i). Hes6 staining was used as a cochlear developmental marker and was colocalized with SSTR5 (Fig. 1f). Primary anti body omission was used as negative control (Fig. 1a, d, g).

Expression and localization of SSTR3, -4 and -5 in the mouse cochlea

We performed RT-PCR to determine SSTR3, -4 and -5 gene expression in the SG, CL and OC of 0-, 5-, 10-, 14- and 21-day-old WT mice. One band of the correct size was observed for every tissue, indicating the presence of mRNA for all three SSTRs. The amplification
Fig. 3 SST3, 4 and 5 receptor immunofluorescence labeling of the adult mouse cochlea. a SSTR3 (red) immunolabeling was detected in the OC and SG. $\mathbf{b}$ SSTR3 was detected in IHC, $\mathrm{OHC}$ and supporting cells Deiter's $(D C)$ and Pillar cells $(P C)$. c SSTR4 was detected in the OC and SG. d SSTR4 immunolabeling was prominent in the supporting cells of $\mathrm{OHC}$ but was also detected in the IHC and OHC. e SSTR5 immunolabeling was seen in the OC and SG. $\mathbf{f}$ SSTR5 was detected in IHC and OHC. Cell nuclei (DAPI) are in blue. Scale bars (a, c, e) $100 \mu \mathrm{m}$, (b, d, f) $50 \mu \mathrm{m}$
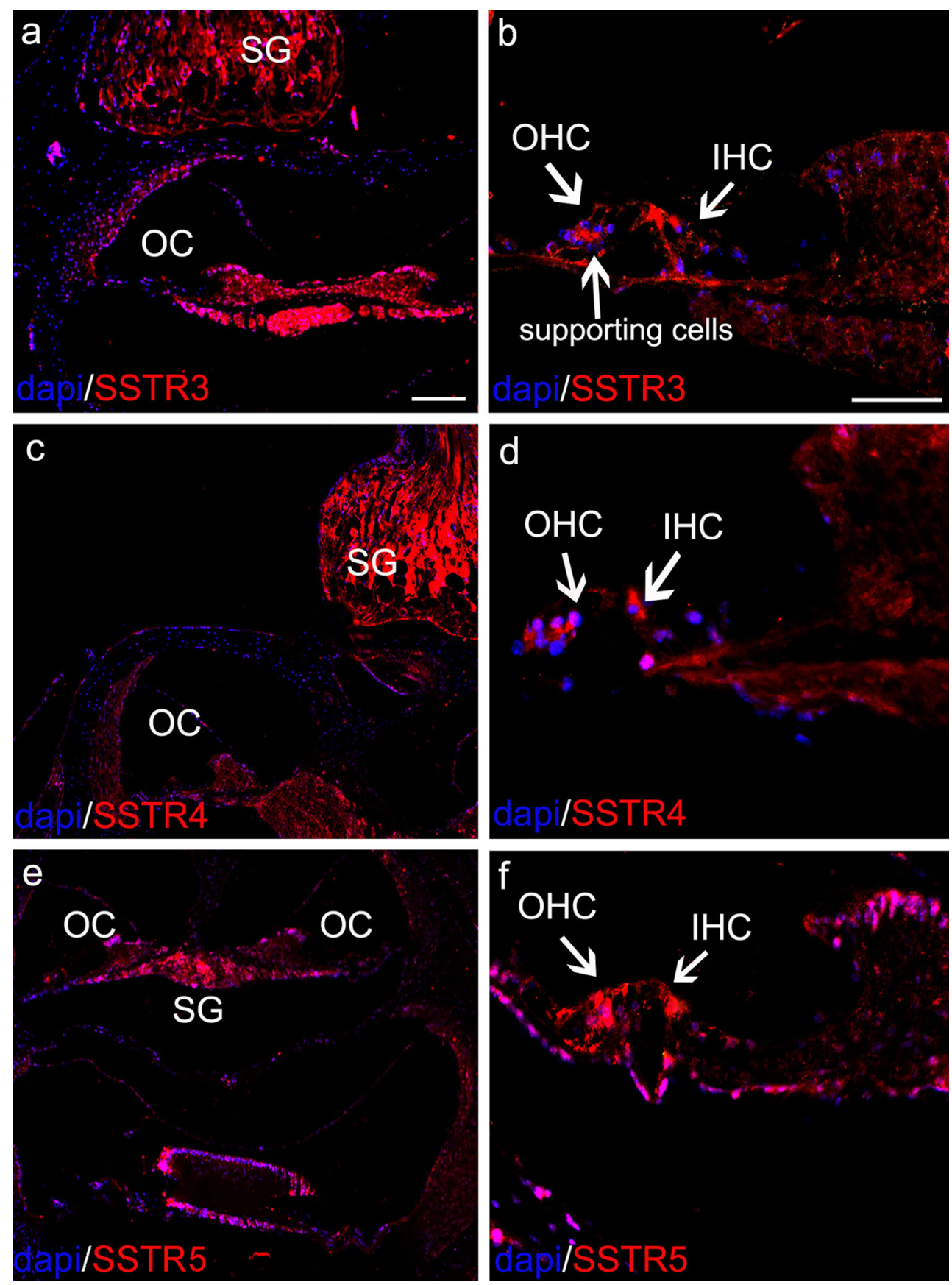

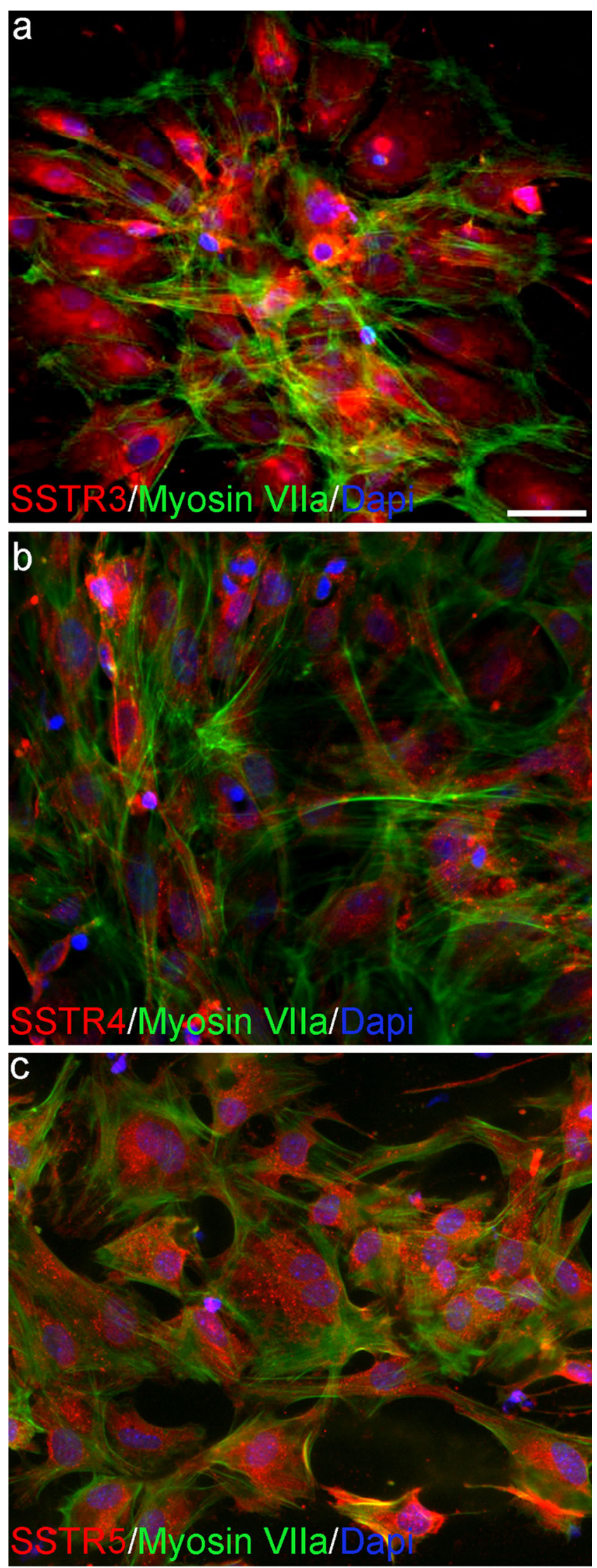

Fig. 4 Immunofluorescence microscopy detection of SSTR3, -4 and -5 in cultivated NEsc from the OC. Cell nuclei are depicted in blue. The cell membranes of cultivated cells are positive for the HC marker myosin VIIa. a Perinuclear localization of SSTR3 (red), b SSTR4 (red), and c SSTR5 (red) were detected. Scale bar (a-c) $100 \mu \mathrm{m}$

of $\beta$-actin confirmed successful cDNA synthesis and cDNA from the brain tissue served as a positive control (Fig. 2). Omission of RT-PCR served as a negative control. During cochlear development, SSTR3, -4 and -5 mRNA were not differentially expressed between different postnatal stages.

Immunolocalization of the SST3, 4 and 5 receptor in adult mouse cochlea

Immunohistochemistry was performed to localize SSTR3, -4 and -5 in the adult mouse cochlea. Prominent immunostaining was detected for SSTR3 in the basal part of OHC, the apical part of IHC Pillar cells and SG cell bodies (Fig. 3a, b). Analysis by fluorescence microscopy revealed localization of SSTR4 in the supporting cells of OHC. A strong signal was also detected at the apical part of IHC (Fig. 3d). The SG exhibited a strong signal in the cell body of ganglion cell types (Fig. 3c). SSTR5 immunoreactivity exhibited an overall distribution in OC with strong immunostaining in SG cells, the apical and basal part of OHC and the apical part of IHC (Fig. 3e, f).

SSTR3, -4 and -5 proteins expression in passaged NEsc derived from cochlea

Immunocytochemistry was performed to localize the three SSTRs in cultured NEsc from the immature cochlea. Myosin VII served as a marker for hair cells and cell nuclei were stained in blue with DAPI. SSTR3, -4 and -5 were detected in the myosin VII-positive NEsc and were mainly localized to the perinuclear region (Fig. 4a-c).

\section{SSTR3, -4 and -5 expressions in DKO cochlea}

In the adult cochlea of DKO mice, the expression of SSTR3 was restricted to IHC and $\mathrm{OHC}$ without positive labeling of supporting cells Deiter's (DC) and Pillar cells (PC) (Fig. 5a, c) in comparison with the expression in the cochlea of WT mice. The SSTR4 expression pattern in the cochlea of DKO mice showed subtle differences from that of WT mice. SSTR4 expression was detected in IHC and OHC but it was localized to the apical part of the hair cells compared to WT mice (Fig. 5d-f). SSTR5 protein was prominent in the IHC and $\mathrm{OHC}$ of DKO cochlea. In 
the cochlea of KO, SSTR5 expression was observed mainly in the supporting cells (Fig. 5h, i).

Comparative analysis of the protein level of SSTR3, -4 and -5 in the cochlea from P7 WT and DKO mice

The expression of all three SSTRs was assessed in brain and in OC explants from WT and DKO mice. We observed a reduced level of SSTR3 in DKO mice compared with WT mice (Fig. 6). No differences in the expression of SSTR4 were found between WT and DKO. The expression level of SSTR5 was higher in DKO mice than in the WT mice (Fig. 6). $\beta$ Actin was used as loading control.

Quantitative comparison of SSTR3,-4 and -5 gene expression in the WT and DKO mice

We performed real-time PCR to determine the quantitative expression level of SSTR3, -4 and -5 in the OC of
14- and 21-day-old WT and DKO mice. The expression of SSTR3 was significantly decreased in OC explants from P14 mice but not from P21 mice compared to agematched OC explants from WT mice (Fig. 7a). The expression of SSTR4 was significantly decreased in the OC explants from P14 and P21 compared to the age-matched OC explants from WT mice (Fig. 7b). The expression of SSTR5 was increased in OC explants from P14 and P21 DKO mice compared to agematched WT mice (Fig. 7c).

\section{Discussion}

We initiated this study to explore the expression of SSTR3-5 in the postnatal and adult mammalian cochlea. To learn more about the regulation of individual SSTR receptors, we analyzed expression levels of SSTR3-5 in SSTR1 and SSRT2 receptor double-KO mice.
Fig. 5 Immunohistochemical localization of SSTR3, 4 and 5 in the cochlea of WT, SSTR1 KO and DKO adult mice. Cell nuclei are in blue. a-c SSTR3 (red) expression in $\mathrm{IHC}$ and $\mathrm{OHC}$ in DKO cochlea compared to WT was reduced but high expression was detected in the supporting cells (DC) and (PC). No difference was seen in expression of SSTR3 between WT and SSTR1 KO mice. $\mathbf{d}-\mathbf{f}$ SSTR4 (red) was not differentially expressed between WT, SSTR1 $\mathrm{KO}$ and DKO mice. $\mathbf{g}-\mathbf{i}$ SSTR5 (red) was over expressed prominently in the IHC as well as supporting cells of DKO cochlea in comparison with WT mouse cochlea. Scale bar(a-i) $100 \mu \mathrm{m}$
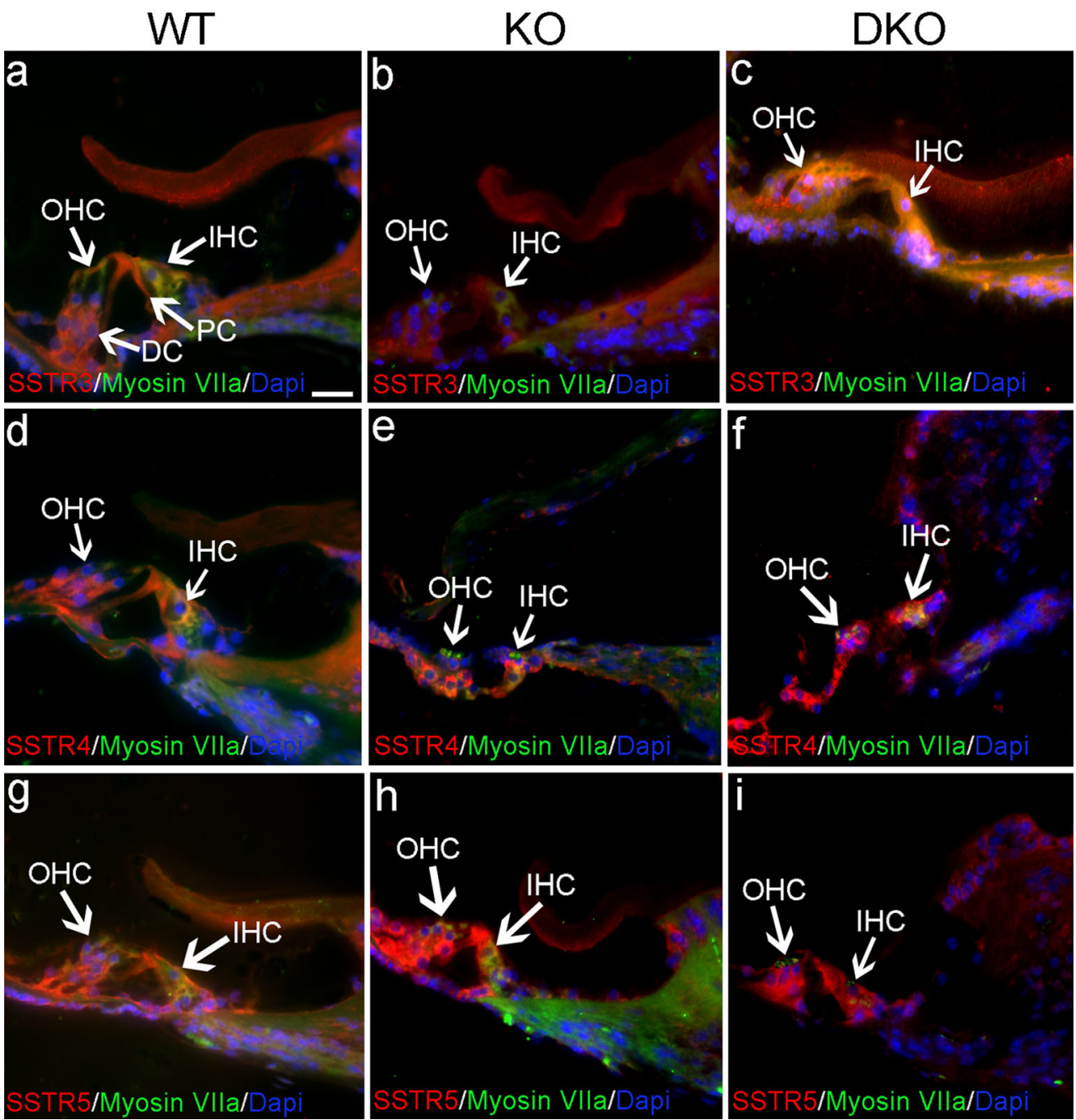
SSTR3 in the mouse cochlea

Using immunohistochemistry, we detected SSTR3 in hair cells as well as in supporting cells of the OC. Interestingly, we observed a significant down-regulation of SSTR3 levels in SSTR1 and SSTR2 DKO mice compared to wild-type mice. In addition, in the DKO mice, we observed SSTR3 expression mainly in hair cells, while in wild-type mice, SSTR3 was mainly expressed in pillar cells. Therefore. we see an altered expression pattern together with a decreased expression level of SSTR3 after SSTR1 and SSTR2 deletion. What might be the role of SSTR3 in the inner ear? To date, we have no information on SSTR3 function within the cochlea. Since deletion of SSTR1 and SSTR2 alters both expression pattern and localization, we can assume that SSTR3 has to some extent redundant functions to SSTR1 and SSTR2. SSTR3 is also expressed in the central nervous system. In the cortex, SSTR3 can primarily be found in the layer V (Hervieu and Emson 1999). In situ studies have also confirmed those findings (Kong et al. 1994; Senaris at al. 1994). It has been speculated that SSTR3 influences cortical excitability (Epelbaum 1986; Epelbaum et al. 1994). Finally, some authors have suggested that SSTR3 might act as a neurotrophic factor (Wulfsen et al. 1993; Yamada et al. 1992).

\section{SSTR4 in the mouse cochlea}

In the cochlea, we found SSTR4 expression in hair cells and pillar cells. Most interestingly, expression of SSTR4 in hair cells was lost in SSTR1 and SSTR2 DKO mice compared to wild-type mice. Similar to SSTR3, SSTR4 mRNA was down-regulated in DKO animals compared to wild-type animals. Therefore, we can also assume that SSTR4 displays to some extent redundant function to SSTR1 and SSTR2. To date, we have no data available on the function of SSTR4 in the mammalian cochlea. In the brain, SSTR4 has been linked to modulation of complex behaviors, including motor activity and memory (Schreff et al. 2000). Also, SSTR4 can mediate regional- and cell-specific postsynaptic responses (Schreff et al. 2000; Schulz et al. 2000).

SSTR5 in the mouse cochlea

SSTR5 was found both in hair cells as well as in supporting cells. Interestingly, in the cochlea of SSTR1 and SSTR2 DKO mice, SSTR5 was up-regulated in hair and supporting cells. Using real-time PCR, we found an up-regulation of SSTR5 in DKO mice compared to wild-type mice. This finding is similar to results of our previous studies that SSTR1 loss has a major effect on the expression of SSTR2 (Radojevic et al. 2011). The function of SSTR5 in the cochlea is currently not known. In the retina, SSTR5 functions as an autoreceptor and localizes to GABAergic cells, including dopaminergic and cholinergic $\mathrm{ACs}$ ( $\mathrm{Ke}$ and Zhong 2007), suggesting that the receptor is involved in protein release. The same regulation mechanism has been found in the striatum, where it is believed that calcium channels are involved (Akopian et al. 2000; Petrucci et al. 2001; Sun et al. 2002; Kouvidi et al. 2006). SRIF stimulation on SSTR5 inhibits L-type or N-type calcium currents and activation of SSTR5 could result in
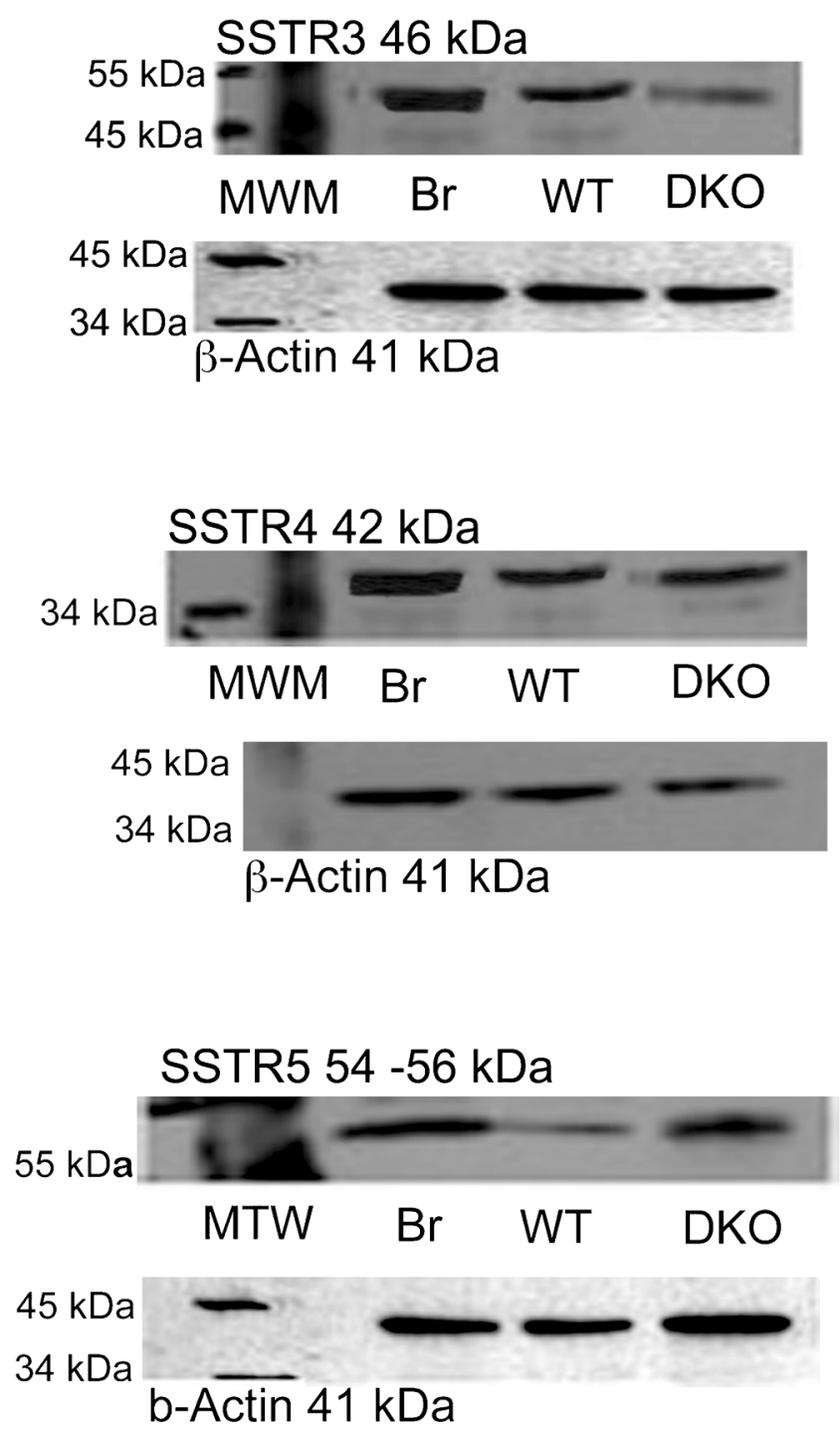

Fig. 6 Detection of SSTR3, -4 and -5 in the OC of WT and DKO mice cochlea by western blotting. Western blotting reveals that SSTR3, -4 and 5 can be detected in protein extracts from WT and DKO OC at P21. Brain extract from WT mice served as positive control. Actin served as a standard to demonstrate that equal amounts of proteins have been loaded. MWM Molecular weight marker 
Fig. 7 The cochlear gene expression of SSTR 3, 4 and 5 in OC explants at $\mathrm{P} 14$ and $\mathrm{P} 21$ from WT and DKO mice. The relative distribution of SSTR3, -4 and -5 mRNA expression in OC tissue from WT and DKO mice of different postnatal ages was quantified by real-time PCR. GAPDH was used as an endogenous control. Gene expression levels are expressed as the mean $( \pm \mathrm{SE})$ fold increase compared to the values obtained in OC explants from P14 and P21 WT and DKO mice. Data were obtained from five independent experiments. ${ }^{*} p<0.002$,

${ }^{* *} p<0.004$ using Student's $t$ test
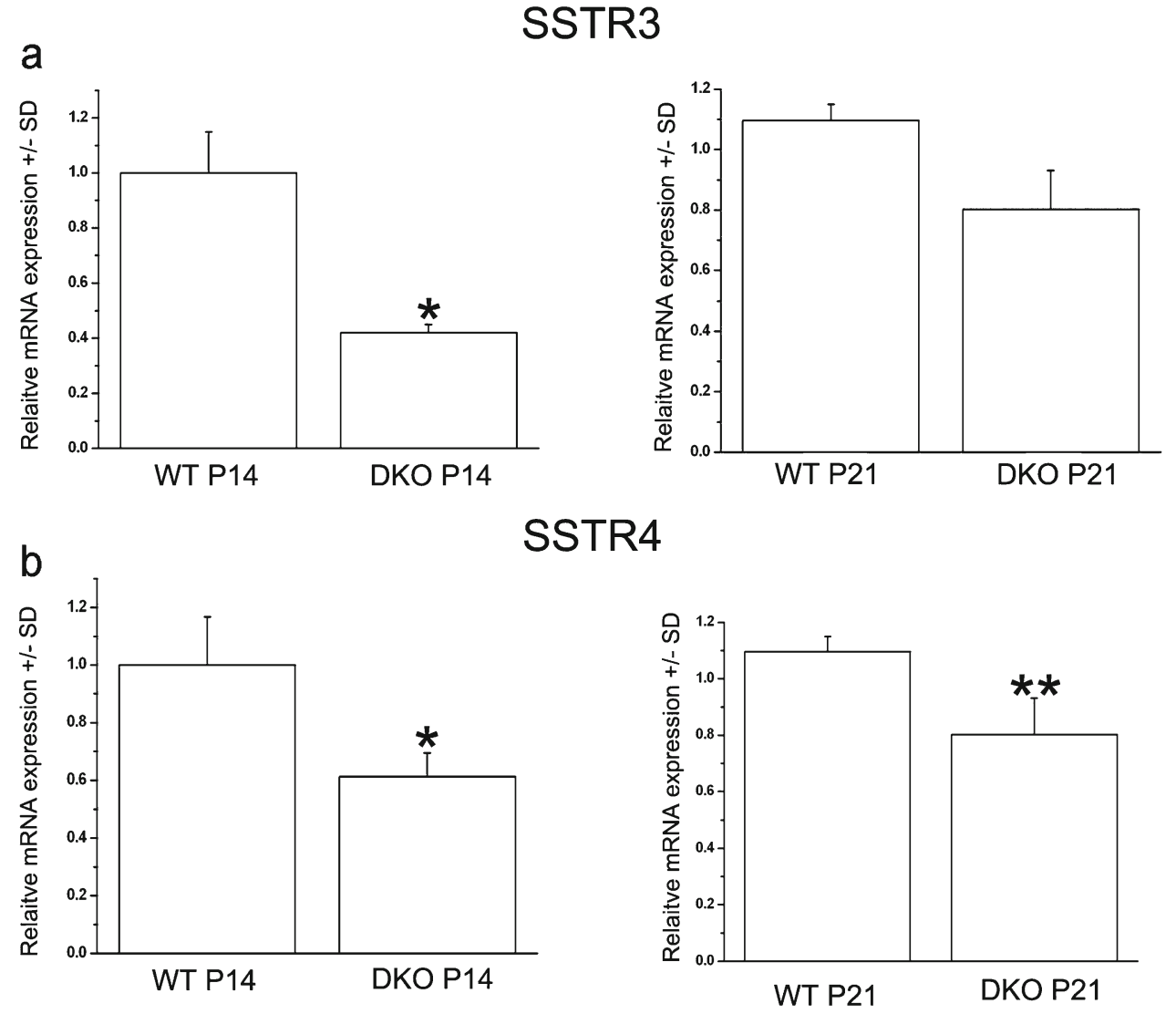

SSTR5

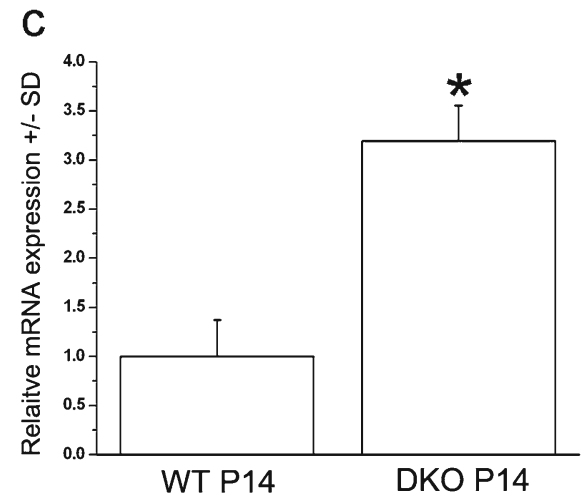

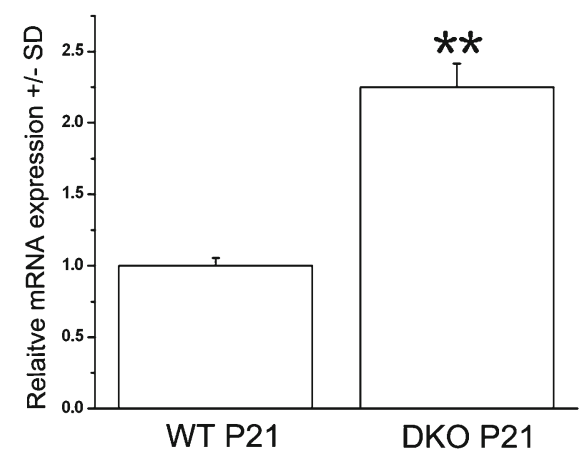

increased intracellular calcium levels (Tallent et al. 1996; Sun et al. 2002; Wilkinson et al. 1997).

Regulation of SSTRs in the cochlea

No major compensatory regulation of SST or individual SSTRs have yet been described in specific brain regions as a consequence of the genetic deletion of SSTR1, SSTR2, or both (Hannon et al. 2002). In contrast, major alterations of the somatostatinergic system as a consequence of SSTR1 or SSTR2 deletion have been demonstrated in the retina (Dal Monte et al. 2003; Casini 2005). In retinas from SSTR KO mice, SSTR1 and SSTR2 receptor expression have been found to compensate for each other; SSTR1 loss resulted in increased expression of SSTR2 (Dal Monte et al. 2003). We found compensatory down-regulation of SSTR3 and SSTR4 and up-regulation of SSTR5 in DKO mice compared to WT mice (Fig. 5). In SSTR1 KO mice, we observed up-regulation of SSTR2 and SSTR5 compared to wild-type mice (Fig. 5). Based on these observations, we can divide SSTRs into two groups: SSTR3 and SSTR4 are down-regulated after SSTR isoform deletion, while SSTR2 and SSTR5 are up-regulated. To date, we do not have an answer to explain this interesting observation. We found that all five SSTR subtypes were 


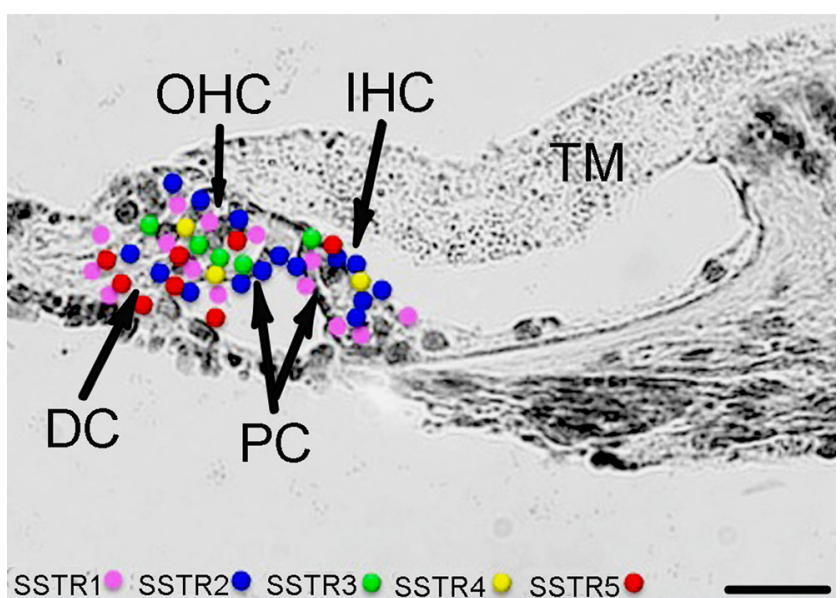

Fig. 8 Summary of the expression patterns of the five SSTRs as seen on a light microscopy image of mouse adult cochlea stained with hematoxylin/eosin. OHC, IHC, DC, PC, tectorial membrane (TM). Scale bar $25 \mu \mathrm{m}$

expressed in the cochlea with considerable regional overlap (Fig. 8). The function of these five receptors in the OC is currently unknown.

\section{Conclusion}

In summary, the present study is the first to describe the expression of SSTR3-5 in cochlear tissue. Together with our previous study (Radojevic et al. 2011), we demonstrated that all five SST receptors are compartmentally expressed in the $\mathrm{OC}$ and SG. The presence of SSTRs within the mammalian cochlea, their specific expression in the $\mathrm{OC}$ and their subtypespecific compensatory regulation as a consequence of distinct SSTR deletion suggest an important role of the somatostatinergic system within the inner ear. These receptors may have crucial functional roles in the inner ear and dysfunction of these receptors may lead to hearing disturbances (Bodmer et al. 2012).

Acknowledgments The authors thank Markus Saxer for technical help. The Schwerhörigenverein Nordwestschweiz supported this work.

\section{References}

Akopian A, Johnson J, Gabriel R, Brecha N, Witkovsky P (2000) Somatostatin modulates voltage-gated $\mathrm{K}(+)$ and $\mathrm{Ca}(2+)$ currents in rod and cone photoreceptors of the salamander retina. J Neurosci 20: 929-936

Beaudet A, Greenspun D, Raelson J, Tannenbaum GS (1995) Patterns of expression ofSSTR1 and SSTR2 somatostatin receptor subtypes in the hypothalamus of the adult rat: relationship to neuroendocrine function. Neuroscience 65:551-561
Blatchley BJ, Cooper WA, Coleman JR (1987) Development of auditory brainstem response to tone pip stimuli in the rat. Brain Res 429:7584

Bodmer D, Brand Y, Radojevic V (2012) Somatostatin Receptor Types 1 and 2 in the Developing Mammalian Cochlea. Dev Neurosci 34: 342-353

Breder CD, Yamada Y, Yasuda K, Seino S, Saper CB, Bell GI (1992) Differential expression of somatostatin receptor subtypes in brain. J Neurosci 12:3920-3934

Casini G (2005) Neuropeptides and retinal development. Arch Ital Biol 143:191-198

Cervia D, Bagnoli P (2007) An update on somatostatin receptor signaling in native systems and new insights on their pathophysiology. Pharmacol Ther 116:322-341

Chen J, Ruan R (2009) Identifying stable reference genes for evaluation of antioxidative enzyme gene expression in auditory cortex and cochlea of young and old Fischer 344 rats. Acta Otolaryngol 129:644-650

Dal Monte M, Petrucci C, Vasilaki A, Cervia D, Grouselle D, Epelbaum J, Kreienkamp HJ, Richter D, Hoyer D, Bagnoli P (2003) Genetic deletion of somatostatin receptor 1 alters somatostatinergic transmission in the mouse retina. Neuropharmacology 45:1080-1092

Epelbaum J (1986) Somatostatin in the central nervous system: physiology and pathological modifications. Prog Neurobiol 27:63-100

Epelbaum J, Dournaud P, Fodor M, Viollet C (1994) The neurobiology of somatostatin. Crit Rev Neurobiol 8:25-44

Hall KL, Pitts DR, Anne S, Semaan MT, Alagramam KN, Megerian CA (2007) Optimization of ribonucleic acid detection from archival Guinea pig temporal bone specimens. Otol Neurotol 28: $116-123$

Hannon JP, Nunn C, Stolz B, Bruns C, Weckbecker G, Lewis I, Troxler T, Hurth K, Hoyer D (2002) Drug design at peptide receptors: somatostatin receptor ligands. J Mol Neurosci 18:15-27

Harrington KA, Schindler M, Humphrey PP, Emson PC (1995) Expression of messenger RNA for somatostatin receptor subtype 4 in adult rat brain. Neurosci Lett 188:17-20

Hervieu G, Emson PC (1999) Visualisation of somatostatin receptor sst(3) in the rat central nervous system. Brain Res Mol Brain Res 71:290-303

Johnson J, Caravelli ML, Brecha NC (2001) Somatostatin inhibits calcium influx into rat rod bipolar cell axonal terminals. Vis Neurosci 18: 101-108

Ke JB, Zhong YM (2007) Expression of somatostatin receptor subtype 5 in rat retinal amacrine cells. Neuroscience 144:1025-1032

Kong H, DePaoli AM, Breder CD, Yasuda K, Bell GI, Reisine T (1994) Differential expression of messenger RNAs for somatostatin receptor subtypes SSTR1, SSTR2 and SSTR3 in adult rat brain: analysis by RNA blotting and in situ hybridization histochemistry. Neuroscience 59:175-184

Kouvidi E, Papadopoulou-Daifoti Z, Thermos K. (2006) Somatostatin modulates dopamine release in rat retina. Neurosci Lett 391:82-86

Kreienkamp HJ, Akgun E, Baumeister H, Meyerhof W, Richter D (1999) Somatostatin receptor subtype 1 modulates basal inhibition of growth hormone release in somatotrophs. FEBS Lett 462:464-466

Patel YC (1999) Somatostatin and its receptor family. Front Neuroendocrinol 20:157-198

Petrucci C, Resta V, Fieni F, Bigiani A, Bagnoli P (2001) Modulation of potassium current and calcium influx by somatostatin in rod bipolar cells isolated from the rabbit retina via sst2 receptors. Naunyn Schmiedebergs Arch Pharmacol 363:680-694

Radojevic V, Hanusek C, Setz C, Brand Y, Kapfhammer JP, Bodmer D (2011) The somatostatinergic system in the mammalian cochlea. BMC Neurosci 12:89

Reichlin S (1983) Somatostatin (second of two parts). N Engl J Med 309: $1556-1563$

Schindler M, Harrington KA, Humphrey PP, Emson PC (1995) Cellular localisation and co-expression of somatostatin receptor messenger RNAs in the human brain. Brain Res Mol Brain Res 34:321-326 
Schreff M, Schulz S, Handel M, Keilhoff G, Braun H, Pereira G, Klutzny M, Schmidt H, Wolf G, Hollt V (2000) Distribution, targeting, and internalization of the sst4 somatostatin receptor in rat brain. J Neurosci 20:3785-3797

Schulz S, Handel M, Schreff M, Schmidt H, Hollt V(2000) Localization of five somatostatin receptors in the rat central nervous system using subtype-specific antibodies. J Physiol 94:259-264

Selmer IS, Schindler M, Humphrey PP, Emson PC (2000) Immunohistochemical localization of the somatostatin sst(4) receptor in rat brain. Neuroscience 98:523-533

Senaris RM, Humphrey PP, Emson PC (1994) Distribution of somatostatin receptors 1, 2 and 3 mRNA in rat brain and pituitary, Eur $\mathrm{J}$ Neurosci 6:1883-1896

Smaili S, Hirata H, Ureshino R, Monteforte PT, Morales AP, Muler ML, Terashima J, Oseki K, Rosenstock TR, Lopes GS, Bincoletto C (2009) Calcium and cell death signaling in neurodegeneration and aging. An Acad Bras Cienc 81:467-475

Stroh T, Kreienkamp HJ, Beaudet A (1999) Immunohistochemical distribution of the somatostatin receptor subtype 5 in the adult rat brain: predominant expression in the basal forebrain. J Comp Neurol 412: 69-82

Sun X, Barnes S, Baldridge WH (2002) Adenosine inhibits calcium channelcurrents via $\mathrm{A} 1$ receptors on salamander retinal ganglion cells in a mini-slice preparation. J Neurochem 81:550-556

Tachibana M, Rothman JM, Guth PS (1979) Somatostatin along the auditory pathway. Hear Res 1:365-368

Takatsuki K, Sakanaka M, Shiosaka S, Inagaki S, Takagi H, Senba E, Hara Y, Kawai Y, Minagawa H, Iida H, Tohyama M (1982)
Pathways and terminal fields of the cochlearofugal somatostatin tracts of very young rats. Brain Res 255:613-626

Tallent M, Liapakis G, O'Carroll A-M, Lolait SJ, Dichter M, Reisine T (1996) Somatostatin receptor subtypes SSTR2 and SSTR5 couple negatively to an L-type $\mathrm{Ca} 2+$ current in the pituitary cell line AtT20. Neuroscience 71:1073-1081

Thermos K (2003) Functional mapping of somatostatin receptors in the retina: a review. Vision Res 43:1805-1815

Thoss VS, Perez J, Duc D, Hoyer D (1995) Embryonic and postnatal mRNA distribution of five somatostatin receptor subtypes in the rat brain. Neuropharmacology 34:1673-1688

Vasilaki A, Thermos K (2009) Somatostatin analogues as therapeutics in retinal disease. Pharmacol Ther 122:324-333

Wilkinson GF, Feniuk W, Humphrey PPA (1997) Characterization of human recombinant somatostatin sst5 receptors mediating activation of phosphoinositide metabolism. Br J Pharmacol 121:91-96

Wulfsen I, Meterhof W, Fehr S, Richter D(1993) Expression patterns of rat somatostatin receptorgenes in pre- and post-natal brain and pituitary. JNeurochem 61:1549-1552

Yamada Y, Post SR, Wang K, Tager HS, Bell GI, Seino S (1992) Cloning and functional characterization of a family of human and mouse somatostatin receptors expressed in brain, gastrointestinal tract, and kidne. Proc Natl Acad Sci U S A 89:251-255

Zheng H, Bailey A, Jiang MH, Honda K, Chen HY, Trumbauer ME, Van der Ploeg LH, Schaeffer JM, Leng G, Smith RG (1997) Somatostatin receptor subtype 2 knockout mice are refractory to growth hormone-negative feedback on arcuate neurons. Mol Endocrinol 11:1709-1717 